\title{
Post T Tauri stars in the solar neighborhood: isolated or members of young associations and moving groups
}

ARTICLE · FEBRUARY 2009

DOI: $10.1063 / 1.3099274$

CITATIONS

2

8 AUTHORS, INCLUDING:

David Montes

Complutense University of Madrid

197 PUBLICATIONS 1,704 CITATIONS

SEE PROFILE

R. Martínez-Arnáiz

Complutense University of Madrid

17 PUBLICATIONS 236 CITATIONS

SEE PROFILE
READS

19

\section{J. López-Santiago}

Complutense University of Madrid

108 PUBLICATIONS 867 CITATIONS

SEE PROFILE

Antonio Frasca

National Institute of Astrophysics

227 PUBLICATIONS 2,022 CITATIONS

SEE PROFILE 


\title{
Post T Tauri stars in the solar neighborhood: isolated or members of young associations and moving groups.
}

\author{
D. Montes*, J. López-Santiago*, R. M. Martínez-Arnáiz*, P. Guillout ${ }^{\dagger}$, \\ A. Klutsch ${ }^{\dagger}$, R. Freire Ferrero ${ }^{\dagger}$, A. Frasca** and E. Marilli** \\ ${ }^{*}$ Universidad Complutense de Madrid, Dpt. de Astrofísica, Facultad de C.C. Físicas, 28040, \\ Madrid, Spain \\ ${ }^{\dagger}$ Observatoire Astronomique, Université de Strasbourg \& CNRS (UMR 7550), 11 rue de \\ l'Université, 67000 Strasbourg, France \\ ** INAF - Osservatorio Astrofisico di Catania, via S. Sofia, 78, 95123 Catania, Italy
}

\begin{abstract}
Post T Tauri stars (PTTS) are late-type stars in the age range between 10 and $100 \mathrm{Myr}$ filling the gap between T Tauri (TTS) and zero-age main sequence phases. This period of evolution remains ambiguous and until now different studies of young stars have failed to find the numbers of PTTS that are expected. In the last years, some PTTS have been identified among the X-ray detected pre-main sequence stars in some star-forming regions. More recently, additional PTTS have been identified in young associations and moving groups ( $\beta$ Pic, TW Hya, Tucana/Horologium, and the AB Dor). However, many isolated PTTS still remain undiscovered. In this contribution, we compiled the PTTS previously identified in the literature, and identified new candidates using the information provided by the high resolution spectra obtained during our surveys of late-type stars possible members to young moving groups, FGK stars in the solar neighborhood, and RasTyc sample. To identify PTTS we applied an age-oriented definition using relative age indicators ( $\mathrm{Li}$ abundance, chromospheric and coronal emission and the kinematics) as well as color-magnitude diagrams and pre-main sequence isochrones.
\end{abstract}

Keywords: Stars: late-type - chromospheres - stellar activity - pre-main sequence PACS: 97.10.-q, 95.85.Kr, 97.10.Ex, 97.10.Jb, 97.10.Ri, 97.10.Tk, 97.21.+a, 98.20.-d

\section{SELECTION BY THE LITHIUM ABUNDANCE $(A g e)$}

The possible PTTS and young stars analysed in this contribution have been selected from our catalog of late-type stars (F to M) members of young stellar kinematic groups ([10]) and the RasTyc sample (cross-correlation of the ROSAT All-Sky Survey (RASS) with the TYCHO catalog, see [1]; [2]). A large number of these stars have been observed by us over the past few years with high resolution spectroscopy ([11]; [3]; [4]; [12]; [8]; [9]; [6]; [5]; [2]). Using the equivalent width of the Li I line at $6707.8 \AA, E W(\mathrm{Li} \mathrm{I})$, determined by us, plus additional values taken from the literature we have selected the stars with $E W(\mathrm{Li} \mathrm{I})$ above or very close to the upper envelope of the Pleiades open cluster ( $\sim 78 \mathrm{Myr}$ ), see Figs. 1 and 2 . The membership in young moving groups (MGs) like the Local Association, AB Dor, $\beta$ Pic, and Tucana/Horologium (Tuc-Hor) is indicated in Fig. 2 with different symbols (see also the $U, V$ diagram in Fig. 3). The PTTS of the sample (stars in the gap intermediate between the TTS and the young cluster stars, adopting the spectroscopic definition proposed by [7]) are listed in Table 1. 


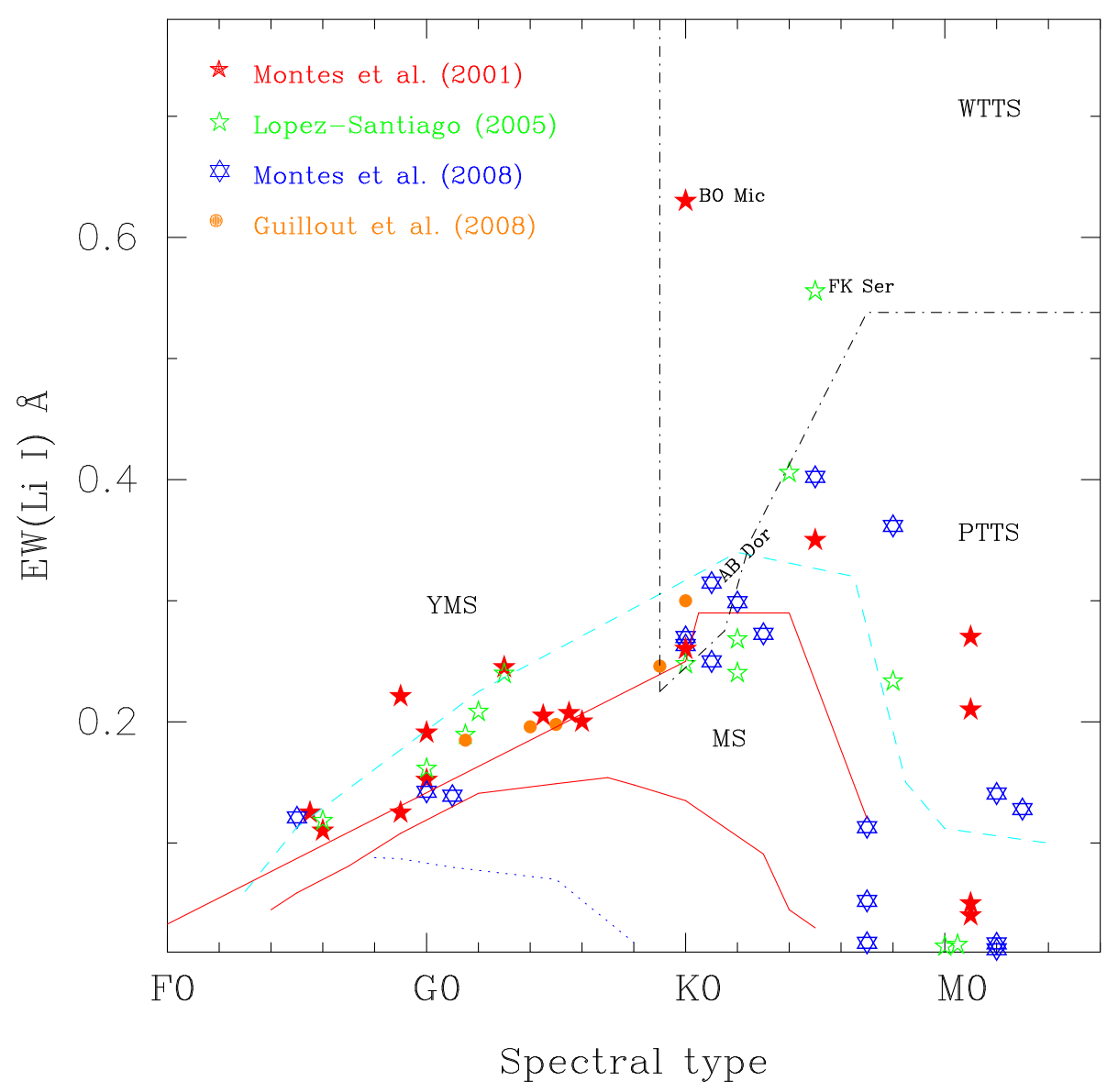

FIGURE 1. Equivalent width of the Li I line at 6707.8 A (from [10]; [11]; [3]; [12]; [2]), as a function of spectral type for the selected stars, compared with the envelopes of well-known stellar clusters (ages from 10 to $600 \mathrm{Myr}$ ). The dot-dashed lines represent the $T_{\text {eff }}$ cutoff at $5250 \mathrm{~K}$ and the $\mathrm{Li}$ isoabundance line for $\log N(\mathrm{Li})=2.8$ (minimum abundance for TTS) used in the PTTS spectroscopic definition by [7].

\section{KINEMATICS OF THE POSSIBLE PTTS}

The kinematics (Galactic space velocity components, $U, V$, see Fig. 3) indicate that all the selected stars are in the region of the young disk stars and very close to the position of the Local Association and other very young stellar associations and moving groups like AB Dor, subgroup B4 of the Local Association, $\beta$ Pic, Tuc-Hor, IC 2391, and Castor (see[10]; [13]; [4]).

\section{ADDITIONAL FGK STARS $(d<25 p c)$}

In our recent survey of FGK stars in the solar neighbourhood $(d<25 \mathrm{pc})$, we have identified additional stars with ages between those of the Pleiades and the Hyades, but any new PTTS (see [8]; [9]; [6]). 


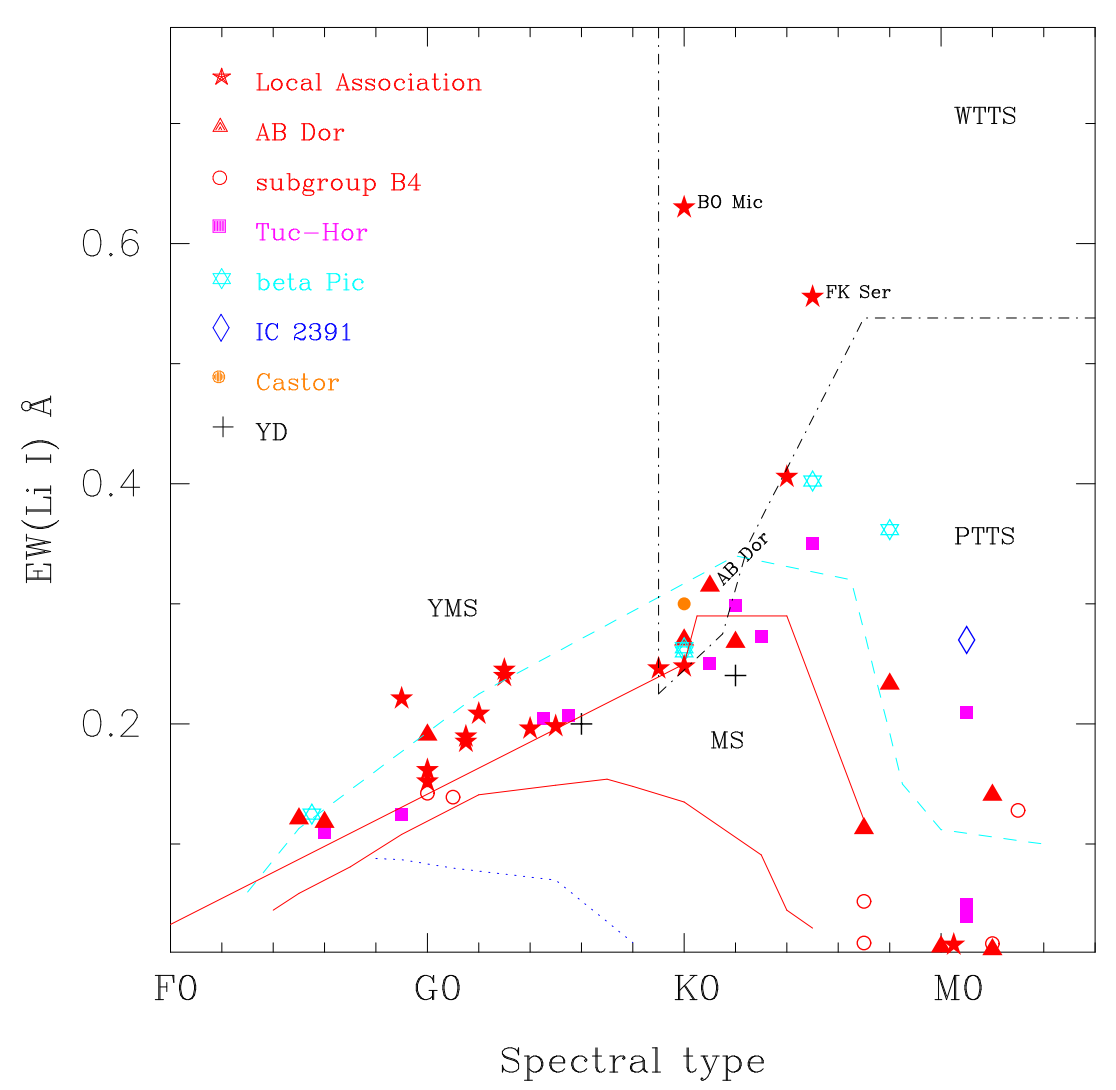

FIGURE 2. As Fig. 1 but in this case different symbols are used to identify the stars members of the Local Association, AB Dor moving group, and B4 subgroup (see [4]), IC2391 and Castor moving groups ([10]), and $\beta$ Pic and Tuc-Hor associations ([13]).

TABLE 1. The PTTS of the sample

\begin{tabular}{lccl}
\hline Name & SpT & $E W(\mathbf{L i}$ I) $(\mathbf{m A ̊})$ & MG \\
\hline BD+45 598 & K0 & 300 & Castor \\
UY Pic & K0 & 270 & AB Dor \\
PZ Tel & K0 & 260 & $\beta$ Pic \\
V343 Nor & K0 & 264 & $\beta$ Pic \\
BD+17 4799 & K0 & 248 & LA \\
AB Dor & K1 & 315 & AB Dor \\
AB Pic & K2 & 299 & Tuc-Hor \\
BD+17 232 & K4 & 406 & LA \\
AO Men & K5 & 402 & $\beta$ Pic \\
HD 3221 & K5 & 350 & Tuc-Hor \\
HD 139751 & K7 & 113 & AB Dor \\
HIP 23309 & K8 & 362 & $\beta$ Pic \\
LO Peg & K8 & 233 & AB Dor \\
HIP 1910 & M0 & 210 & Tuc-Hor \\
V1005 Ori & M1 & 270 & IC2391 \\
GSC8894-426 & M2 & 141 & AB Dor \\
HIP 17695 & M3 & 128 & B4 \\
\hline
\end{tabular}




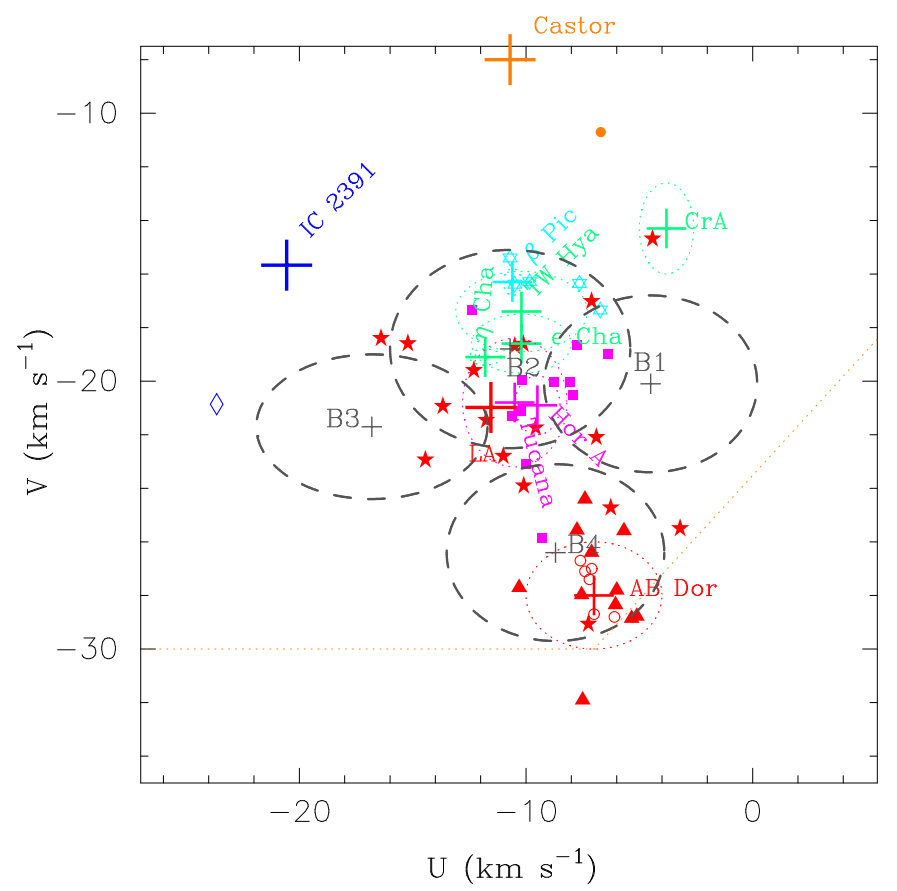

FIGURE 3. Position in the $(U, V)$-plane of the selected stars as possible PTTS. Symbols as in Fig. 2.

\section{ACKNOWLEDGMENTS}

This work was supported the Spanish Ministerio de Educación y Ciencia (MEC), Programa Nacional de Astronomía y Astrofísica under grants AYA2005-02750 and AYA2005-00954, and the "Comunidad de Madrid" under PRICIT projects S-0505/ESP0237 (ASTROCAM) and S-0505/ESP/000361 (ASTRID).

\section{REFERENCES}

1. Guillout, P., Schmitt, J. H. M. M., Egret, D., et al. 1999, A\&A, 351, 1003

2. Guillout, P., Klutsch, A., Frasca, A., et al. 2008, A\&A, in preparation

3. López-Santiago, J. 2005, PhD, UCM

4. López-Santiago, J., Montes, D., Crespo-Chacón, I., \& Fernández-Figueroa, M. J. 2006, ApJ, 643, 1160

5. Klutsch, A., Guillout, P., Frasca, A., et al. 2008, The 14th Cool Stars, Stellar Systems and the Sun workshop, van Belle, G., ed., ASP conf. Ser., V. 384

6. Maldonado, J., Martínez-Arnáiz, R. M., Eiroa, C., Montes, D. 2008, The 15th Cool Stars, Stellar Systems and the Sun workshop, AIP Conference Proceedings Series, this volume

7. Martín, E. L. 1997, A\&A, 321, 492

8. Martínez-Arnáiz, R. M., Maldonado, J., Montes, D., et al. 2008, The 14th Cool Stars, Stellar Systems and the Sun workshop, van Belle, G., ed., ASP conf. Ser., V. 384

9. Martínez-Arnáiz, R. M., Maldonado, J., Montes, D., et al. 2008, The 15th Cool Stars, Stellar Systems and the Sun workshop, AIP Conference Proceedings Series, this volume

10. Montes, D., López-Santiago, J., Gálvez, M. C., et al. 2001, MNRAS, 328, 45

11. Montes, D., López-Santiago, J., Fernández-Figueroa, M. J. et al. 2001, A\&A, 379, 976

12. Montes, D., López-Santiago, J., Crespo-Chacón, I., et al. 2008, The 14th Cool Stars, Stellar Systems and the Sun workshop, van Belle, G., ed., ASP conf. Ser., V. 384

13. Zuckerman, B., \& Song, I. 2004, ARA\&A, 42, 685 